\title{
Effect of cylindrical geometry on the wet thermal oxidation of AlAs
}

\author{
A. C. Alonzo, X. -C. Cheng, and T. C. McGill ${ }^{\mathrm{a}}$ \\ T. J. Watson Sr. Laboratory of Applied Physics, California Institute of Technology, Pasadena, \\ California 91125
}

(Received 22 December 1997; accepted for publication 21 September 1998)

\begin{abstract}
We have investigated the wet thermal oxidation of AlAs in cylindrical geometry, a typical configuration for vertical-cavity surface-emitting lasers. Through both experiment and theoretical calculations, we demonstrate a significantly different time dependence for circular mesas from what has been reported in the literature both in studies of stripes and in a study of circular mesas. We attribute this different time dependence to the effect of geometry on the oxidation. (C) 1998 American Institute of Physics. [S0021-8979(98)06824-8]
\end{abstract}

\section{INTRODUCTION}

Since Holonyak's group demonstrated a stable oxide of AlAs, formed by wet thermal oxidation, ${ }^{1}$ there has been much interest in using this technique to create a native oxide for the III-V material system. Although recent research has cast doubts on the feasibility of using this oxide for a metaloxide-semiconductor (MOS)-type device, ${ }^{2}$ it has found wide usage in the creation of vertical-cavity surface-emitting lasers (VCSELs). The oxide provides both current confinement and index guiding, producing the lowest threshold ${ }^{3}$ and highest efficiency ${ }^{4}$ VSCELs ever reported.

Although VCSELs frequently employ circular mesas, most of the oxidation rate studies to date have been conducted with stripes. ${ }^{5-9}$ In this article, we describe a model for the oxidation of circular mesas. Our model differs from one previously reported, ${ }^{10}$ and we believe it more accurately includes the effect of cylindrical geometry upon the oxidation process.

\section{BACKGROUND}

The model widely accepted for the wet thermal oxidation of stripes containing buried AlAs layers is based on Grove and Deal's model for the oxidation of silicon. ${ }^{11}$ This model assumes a steady state process so that the flux of oxidant molecules is constant in three components of the oxidation: (1) transport of oxidant molecules to the edge of the mesa; (2) diffusion of oxidant molecules through the already formed oxide; and (3) reaction at the oxidesemiconductor interface. According to this model, the oxidation time can be described as

$$
t=\frac{x_{o}^{2}}{B}+\frac{x_{o}}{B / A},
$$

where we have assumed no initial oxide so that $x_{o}$, the length of oxide formed, is also the position of the oxidesemiconductor interface. In Eq. (1), $B$ is proportional to the diffusion constant for the reactant traveling through the oxide and $B / A$ is a function of both the supply of water vapor at the edge of the mesa and the reaction rate constant for

a)Electronic mail: tcm@ssdp.caltech.edu oxidation at the oxide-semiconductor interface. From this model, a linear oxidation is thought to represent a reactionrate-limited process, while an oxidation length which is proportional to the square root of time is believed to indicate a diffusion-limited process. ${ }^{7}$

\section{THEORY}

In the case of circular mesas, the surface area of the interface changes as the oxidation proceeds, effectively changing the geometry of the oxidation. Therefore, we do not believe that the steady state assumption is valid for the oxidation of circles. As noted by Ochiai et al., ${ }^{7}$ the functional form for the oxidation of stripes, Eq. (1), is composed of a diffusion and a reaction component. The model described in this article treats the movement of the oxidation front in terms of these processes: diffusion of the reactant to the oxide-semiconductor interface and reaction at the interface. We assume that there is an adequate supply of the reactant at the edge of the mesa and that the removal of As-containing products is not the rate-limiting process. We take the oxidation time to be the sum of the time required for reactant atoms to diffuse to the oxide-semiconductor interface and the time required for the atoms to react at that interface:

$$
t_{\text {oxidation }}\left(x_{o}\right)=t_{\text {diffusion }}\left(x_{o}\right)+t_{\text {reaction }}\left(x_{o}\right) \text {. }
$$

Our model, as described by Eq. (2), can be applied to the oxidation of stripes or circles. The functional form we obtain for stripes is the same as that of Grove and Deal's model, Eq. (1). However, when our model is applied to the oxidation of circular mesas, we obtain a functional form which differs significantly from that obtained by applying the steady state formalism to both stripes ${ }^{11}$ and circular mesas. ${ }^{10}$ We believe that, because it is not constrained by the steady state assumption, our model accounts more fully for the influence of cylindrical geometry upon the oxidation process.

\section{A. Theoretical reaction time}

First, we consider the reaction time by writing an expression for the number of atoms reacted in a time $d t_{\text {reaction }}$ :

$R d t_{\text {reaction }}=\rho d V$, 

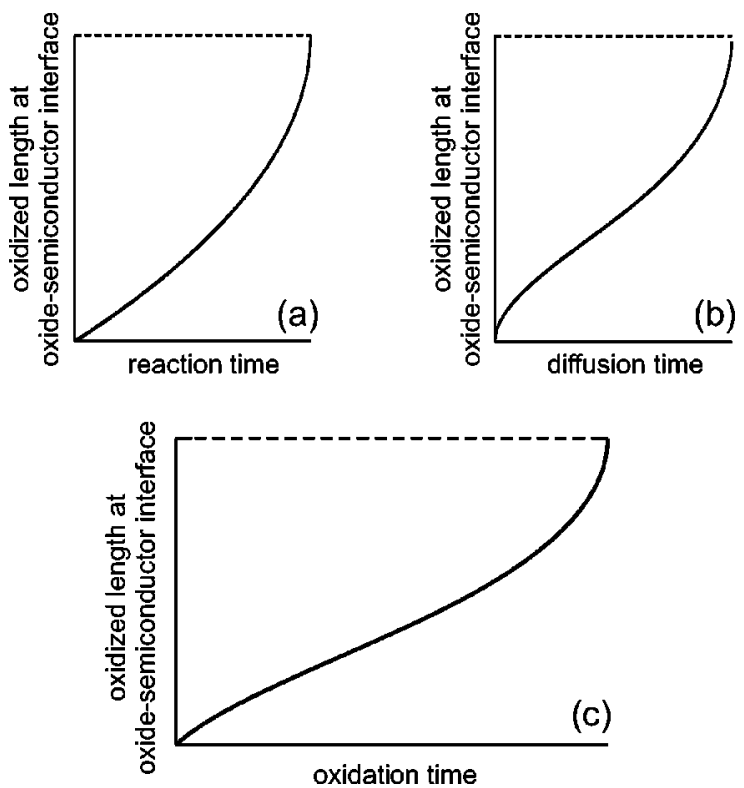

FIG. 1. (a) Functional form for the reaction of oxygen atoms to move the oxide-semiconductor interface in the wet thermal oxidation of AlAs. (b) Functional form for the diffusion of oxygen atoms to the oxidesemiconductor interface in the wet thermal oxidation of AlAs. (c) Functional form for the wet thermal oxidation of AlAs. The dashed line in each plot indicates the oxidized length for complete oxidation (the radius of the mesa).

where $R$ is the reaction rate (in units of atoms per unit time), $\rho$ is the concentration of oxidant molecules in the oxide, and $d V$ is the volume of oxide which must react to move the oxidation front by a differential unit of length. In rectangular coordinates, $d V=L H d x$, where $L$ is the length of the stripe and $H$ is the thickness of the oxidizing layer, while $d V$ $=-2 \pi H r d r$ in cylindrical coordinates. Next, we define a rate $K=R / \rho \sigma$, where $\sigma$ is the outside surface area of the mesa. Therefore, Eq. (3) becomes

$$
K d t=d x
$$

in rectangular coordinates and

$$
K d t=-\frac{r}{a} d x
$$

in cylindrical coordinates, where $a$ is the radius of the mesa. Equations (4) and (5) are subject to the boundary condition that no oxide has been formed at $t_{\text {reaction }}=0$. This condition can be expressed as $x\left(t_{\text {reaction }}=0\right)=0$ in rectangular coordinates and as $r\left(t_{\text {reaction }}=0\right)=a$ in cylindrical coordinates. Integration and application of the boundary condition in rectangular coordinates yield an expression for the oxidized length, $x_{o}$ :

$$
x_{o}=K t_{\text {reaction }} \text {. }
$$

In cylindrical coordinates, we obtain

$$
x_{o}=a-\sqrt{a^{2}-2 a K t_{\text {reaction }}},
$$

where $x_{o}=a-r$. This curve is shown in Fig. 1(a).

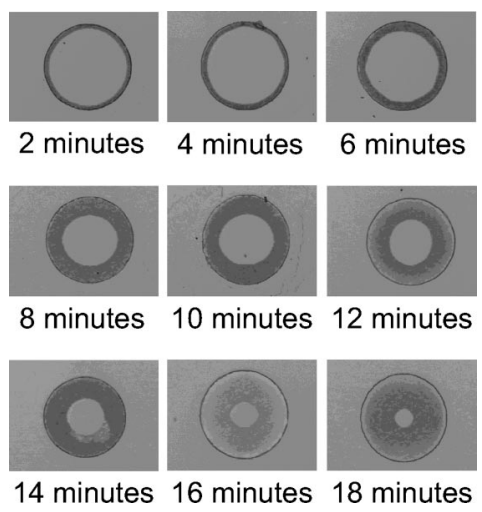

FIG. 2. Optical microscope images of $150 \mu \mathrm{m}$ diameter mesas, after wet thermal oxidation at $425^{\circ} \mathrm{C}$ for the indicated times. The oxidized AlAs layer is viewed through $150 \mathrm{~nm} \mathrm{GaAs}$ and $15 \mathrm{~nm} \mathrm{Al}_{0.3} \mathrm{Ga}_{0.7} \mathrm{As}$.

\section{B. Theoretical diffusion time}

Now considering the diffusion time, we assume that the oxidation front (as seen in Fig. 2) occurs where the concentration of oxygen is half that at the edge of the mesa. Choosing some other fraction does not change the functional form of the solution ${ }^{12}$ but does affect the absolute value of the diffusion constant obtained for the oxidation. We start with the diffusion equation in rectangular coordinates (assuming only diffusion perpendicular to the oxide-semiconductor interface). Next, the following boundary conditions are applied:

(1) There is no oxygen inside the mesa when the oxidation begins.

(2) There is a constant concentration, $C_{\mathrm{O}}$, of oxygen at the edge of the mesa at all times.

In rectangular coordinates, the solution to the diffusion equation with these boundary conditions is

$$
C(x, t)=C_{\mathrm{O}}\left[1-\operatorname{Erf}\left(\frac{x_{o}}{2 \sqrt{D t_{\text {diffusion }}}}\right)\right],
$$

where $D$ is the diffusion constant for oxygen in the oxide film. In order to obtain $x_{o}\left(t_{\text {diffusion }}\right)$, we set Eq. (8) equal to $0.5 C_{\mathrm{O}}$, which yields

$$
x_{o}=\sqrt{k_{D} t_{\text {diffusion}}},
$$

where

$$
k_{D}=4 D\left[\operatorname{Erf}^{-1}(0.5)\right]^{2} .
$$

In cylindrical coordinates, the diffusion equation can be written as ${ }^{13}$

$$
\frac{\partial C}{\partial t_{\text {diffusion }}}=\frac{1}{r} \frac{\partial}{\partial r}\left(r D \frac{\partial C}{\partial r}\right),
$$

where $C$ is the oxygen concentration in the mesa. The boundary conditions can be written as: (1) $C(t=0, r \leqslant a)=0$ and (2) $C(r=a)=C_{\mathrm{O}}$. Carslaw and Jaeger give a solution to this problem: ${ }^{14}$

$$
C\left(r, t_{\text {diffusion }}\right)=C_{\mathrm{O}}\left[1-\frac{2}{a} \sum_{n=1}^{\infty} e^{-D \alpha_{n}^{2} t_{\text {diffusion }}} \frac{J_{0}\left(r \alpha_{n}\right)}{\alpha_{n} J_{1}\left(a \alpha_{n}\right)}\right],
$$


where $J_{n}$ represents the $n$ th-order Bessel function and the $\left\{\alpha_{n}\right\}$ s are solutions to $J_{0}\left(a \alpha_{n}\right)=0$. We numerically solve the equation $C\left(r, t_{\text {diffusion }}\right)=0.5 C_{\mathrm{O}}$ to obtain an expression for the radius of the oxidation front (and thus the oxidized length) as a function of the diffusion time. The resulting curve is plotted in Fig. 1(b).

\section{Oxidation in cylindrical geometry}

Following Eq. (2), our oxidation model is completed by summing the diffusion and reaction times for a given oxidation length, $x_{o}$. The resulting curve for cylindrical coordinates is plotted in Fig. 1(c). It is clear from this curve that the oxidation of circular mesas is predicted to proceed in a much different manner than that of stripes. While the oxidation rate for steady state models (both circles ${ }^{10}$ and stripes ${ }^{11}$ ) decreases as the oxidation proceeds, our model predicts that the oxidation rate will increase for cylindrical geometry. In addition, we would like to point out that a similar effect is both expected and observed for square mesas, ${ }^{15}$ another typical VCSEL configuration. In both circles and squares, the surface area of the oxidation front decreases as the oxidation proceeds, and this geometric effect causes an increase in the oxidation rate.

\section{EXPERIMENT}

\section{A. Sample preparation and data collection}

The samples used in this study were grown on a (100) GaAs substrate in a Perkin Elmer molecular beam epitaxy (MBE) machine. The layer to be oxidized was $100 \mathrm{~nm}$ of $\mathrm{AlAs}$, surrounded on either side by $15 \mathrm{~nm}$ of $\mathrm{Al}_{0.3} \mathrm{Ga}_{0.7} \mathrm{As}$. The structure was capped with $150 \mathrm{~nm}$ of GaAs. Circles, 150 $\mu \mathrm{m}$ in diameter, were defined using standard photolithography procedures. Hardened photoresist served as an etch mask when the samples were placed in 1:8:80 $\mathrm{H}_{2} \mathrm{SO}_{4}: \mathrm{H}_{2} \mathrm{O}_{2}: \mathrm{H}_{2} \mathrm{O}$ to remove the material around the mesas, exposing the AlAs layer. Each sample was etched immediately prior to being inserted into a $425^{\circ} \mathrm{C}$ furnace which was connected to a $90^{\circ} \mathrm{C}$ water bubbler through which $1 \ell /$ min ultrapure nitrogen was flowing. An optical microscope and charge coupled device (CCD) camera were used to obtain images of the mesas. The pictures were then inserted into a drawing program to measure the diameter of the unoxidized portion of each mesa. Sample pictures are shown in Fig. 2.

For each oxidation time, an average of two samples were oxidized in different runs. Each sample contained approximately 30 circles, so each data point represents information from about 60 mesas. There was no systematic difference in oxidation rates for samples oxidized on different days. The data is shown in Fig. 3.

\section{B. Results}

We start by noting that the qualitative behavior of our data is quite different from that expected for stripes, particularly for longer oxidation times. The oxidation rate increases as the oxidation front nears the center of the mesa, in agreement with our model, as shown in Fig. 1(c).

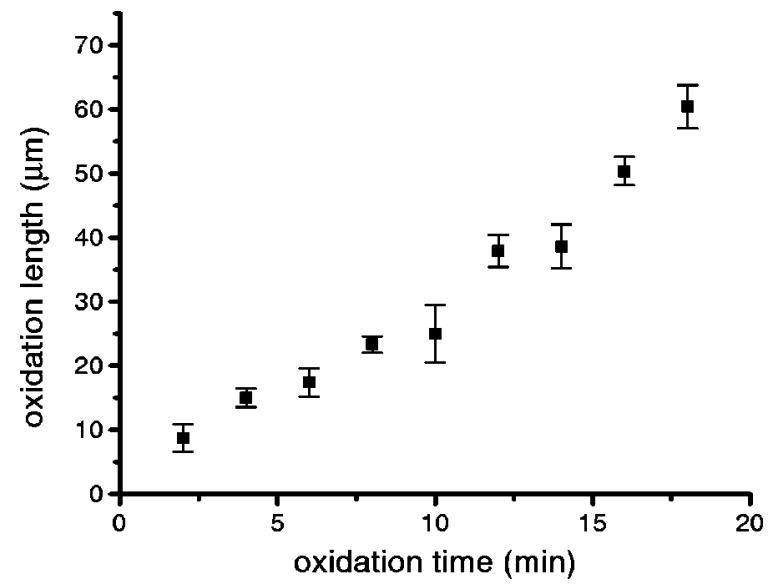

FIG. 3. Measured lateral oxidation length as a function of time for $150 \mu \mathrm{m}$ diameter mesas oxidized at $425^{\circ} \mathrm{C}$.

In order to compare our data and theory, we fit the data to

$$
t_{\text {oxidation }}=t_{\text {diffusion }}\left(D, x_{o}\right)+t_{\text {reaction }}\left(K, x_{o}\right),
$$

using $D$ and $K$ as fitting parameters. The resulting curve is shown in Fig. 4, fit using $D=98 \pm 4 \mu \mathrm{m}^{2} / \mathrm{min}$ and $K=5.3$ $\pm 0.3 \mu \mathrm{m} / \mathrm{min}$.

\section{Comparison to oxidation of stripes}

Figure 5 shows data for the oxidation of stripes under the same conditions $\left(425^{\circ} \mathrm{C}\right.$ furnace, $90^{\circ} \mathrm{C}$ water bubbler, $1 \ell / \mathrm{min}_{2}$ flow) as those used to oxidize the circular mesas. Theoretical curves for circles and stripes are also shown. It is clear from Fig. 5 that the oxidation of circles proceeds much faster than that of stripes, particularly for longer times. In order to plot the curve for stripes, we use Eq. (2), with expressions for $t_{\text {reaction }}$ from Eq. (6) and for $t_{\text {diffusion }}$ from Eq. (9):

$$
t=\frac{x_{o}}{K}+\frac{x_{o}^{2}}{k_{D}}
$$

with $D=98 \mu \mathrm{m}^{2} / \mathrm{min}$. We obtain $K=3.9 \pm 0.2 \mu \mathrm{m} / \mathrm{min}$, which is in reasonable agreement with the value obtained for

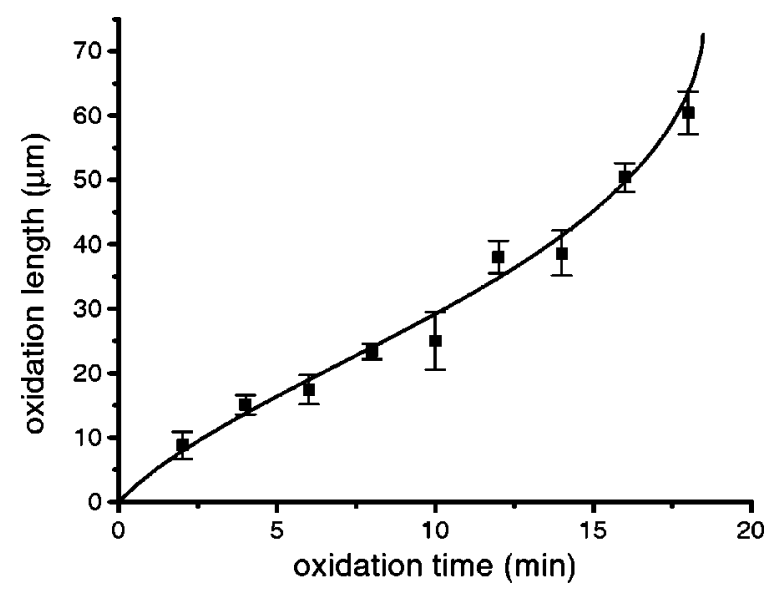

FIG. 4. Model and experimental data for the wet thermal oxidation of AlAs at $425^{\circ} \mathrm{C}$, with $D=98 \mu \mathrm{m}^{2} / \mathrm{min}$ and $K=5.3 \mu \mathrm{m} / \mathrm{min}$. 


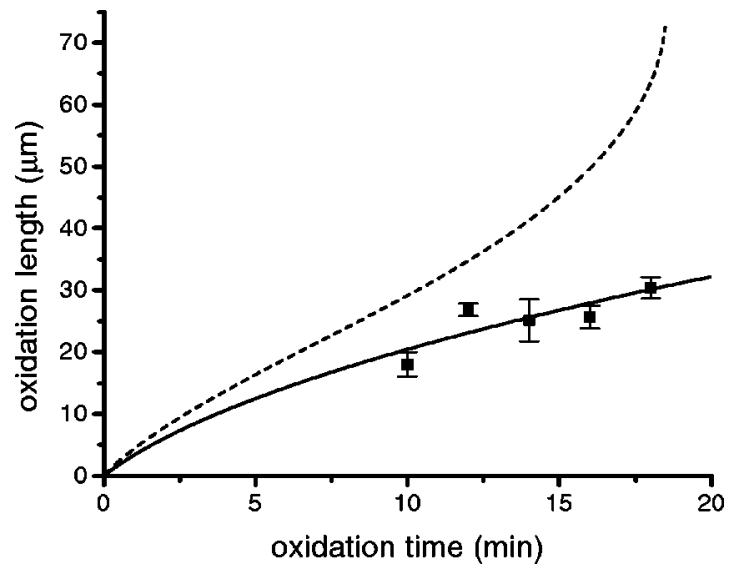

FIG. 5. Measured lateral oxidation length as a function of time for stripes oxidized at $425{ }^{\circ} \mathrm{C}$ shown with theoretical curves for both stripes and circular mesas with $D=98 \mu \mathrm{m}^{2} / \mathrm{min}$. The solid line indicates theory for stripes with $K=3.9 \mu \mathrm{m} / \mathrm{min}$ and the dashed line indicates theory for circular mesas with $K=5.3 \mu \mathrm{m} / \mathrm{min}$.

circles. We would expect both $D$ and $K$ to show agreement between circles and stripes since the oxide's resistance to the diffusion of oxygen and the reactivity of AlAs with oxygen should not be influenced by geometry.

We can use this data to compare our experimental results to those obtained by other researchers for studies involving stripes by comparing Eq. (14) with Grove and Deal's model in Eq. (1). In Grove and Deal's model, $k_{D}=B$. We can use Eq. (10) to calculate $k_{D}$ for our model with $D$ $=98 \mu \mathrm{m}^{2} / \mathrm{min}$, which yields $k_{D}=89 \mu \mathrm{m}^{2} / \mathrm{min}$. A study by Naone et al. ${ }^{16}$ found $B=59 \mu \mathrm{m}^{2} / \mathrm{min}$ for oxidation of AlAs at $425^{\circ} \mathrm{C}$ with a $90{ }^{\circ} \mathrm{C}$ water bubbler and $0.6 \ell / \min N_{2}$ flow. Extracting data from Fig. 4 of Ref. 7 yields $B$ on the order of $100 \mu \mathrm{m}^{2} / \mathrm{min}$ for oxidation of AlAs at $425^{\circ} \mathrm{C}$ with an $85^{\circ} \mathrm{C}$ water bubbler and $0.8 \ell / \mathrm{min} \mathrm{N}_{2}$ flow. Therefore, the $k_{D}$ we obtained for both stripes and circles is in reasonable agreement with values obtained by other researchers for stripes.

Similarly, we can compare the value of $K$ we obtained for circles and stripes with that obtained by other researchers. In Grove and Deal's model, $K=B / A$. Naone et al. ${ }^{16}$ report $B / A=1.1 \mu \mathrm{m} / \mathrm{min}$ and data extracted from Ochiai's paper ${ }^{7}$ indicates $B / A=6.7 \mu \mathrm{m} / \mathrm{min}$. Our values of 5.3 and $3.9 \mu \mathrm{m} /$ min fall between these values.

As shown by other researchers, ${ }^{7,11}$ the oxidation of stripes is reaction rate limited for short times and diffusion limited for longer times. We would expect the same behavior for circular mesas, despite the different time dependence of the oxidation. The reaction and diffusion components of the oxidation of circular mesas are shown in Fig. 6. We can see that, for oxides thinner than $20 \mu \mathrm{m}$, the reaction is the slower process contributing to the oxidation; but, after $20 \mu \mathrm{m}$ of oxide has been formed, diffusion of atoms to the interface takes longer than their reaction once at the interface. Our oxidation of stripes also exhibits a transition between reaction and diffusion processes around the same oxidized length. In addition, as observed in the case of the oxidation of stripes, we see that the oxidation curve has a time dependence similar to that of the reaction process for small times

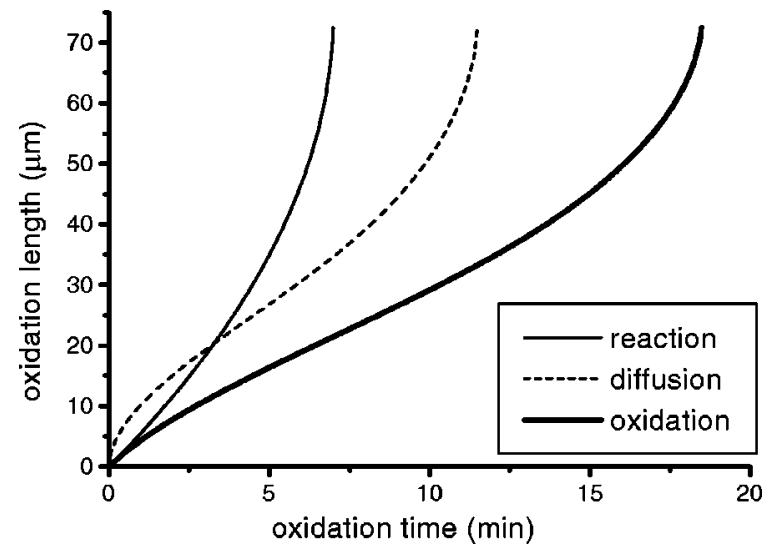

FIG. 6. Reaction curve (light solid line) for $K=5.3 \mu \mathrm{m} / \mathrm{min}$, diffusion curve (dashed line) for $D=98 \mu \mathrm{m}^{2} / \mathrm{min}$, and the resulting oxidation curve (heavy solid line) for circular mesas.

and a time dependence similar to that of the diffusion process for longer times.

As a final comparison to the oxidation of stripes, we believe that the model for the oxidation of circles should reduce to the model for the oxidation of stripes in the limit $a \gg x_{o}$. In this limit, our model reduces exactly to the form for the oxidation of stripes.

\section{CONCLUSIONS}

As demonstrated in this article, the oxidation of circular mesas exhibits a significantly different time dependence than that observed for stripes. Although it is possible to determine the appropriate oxidation time for a given VCSEL structure by trial and error, this method becomes difficult for more complicated structures, such as VCSELs with both oxidized Bragg reflectors and oxidized current apertures. Using oxidation rates obtained for stripes will result in overoxidation, particularly for longer oxidation times. We have also shown that, while the cylindrical geometry has a significant effect on the time dependence of the oxidation process, the basic physics of the oxidation remains unchanged. We hope that the different behavior exhibited by the cylindrical mesas will allow for greater understanding of the wet thermal oxidation process.

\section{ACKNOWLEDGMENTS}

The authors are grateful to T. F. Kuech for suggesting the possible role of cylindrical geometry. The authors also wish to thank J. O. McCauldin and E. T. Yu for useful discussions about the oxidation process. The work was supported in part by the Office of Naval Research under Grant No. N00014-89-J-1141.

${ }^{1}$ J. Dallesasse, J. N. Holonyak, A. Sugg, T. Richard, and N. El-Zein, Appl. Phys. Lett. 57, 2844 (1990).

${ }^{2}$ C. Ashby, J. Sullivan, P. Newcomer, N. Missert, H. Hou, B. Hammons, M. Hafich, and A. Baca, Appl. Phys. Lett. 70, 2443 (1997).

${ }^{3}$ G. Yang, M. MacDougal, and P. Dapkus, Electron. Lett. 31, 886 (1995).

${ }^{4}$ K. Lear, K. Choquette, J. R. P. Schneider, S. Kilcoyne, and K. Geib, Electron. Lett. 31, 208 (1995).

${ }^{5}$ R. Burton and T. Schlesinger, J. Appl. Phys. 76, 5503 (1994).

${ }^{6}$ H. Nickel, J. Appl. Phys. 78, 5201 (1995). 
${ }^{7}$ M. Ochiai, G. Giudice, H. Temkin, J. Scott, and T. Cockerill, Appl. Phys. Lett. 68, 1898 (1996).

${ }^{8}$ F. Kish, S. Maranowski, G. Hofler, J. N. Holonyak, S. Caracci, J. Dallesasse, and K. Hsieh, Appl. Phys. Lett. 60, 3165 (1992).

${ }^{9}$ J.-H. Kim, D. Lim, K. Kim, G. Yang, K. Lim, and H. Lee, Appl. Phys. Lett. 69, 3357 (1996).

${ }^{10}$ B. Koley, M. Dagenais, R. Jin, J. Pham, G. Simonis, G. McLane, and D. Stone, J. Appl. Phys. 82, 4586 (1997).

${ }^{11}$ B. Deal and A. Grove, J. Appl. Phys. 36, 3770 (1965).
${ }^{12}$ D. Cohen, discussion, 1997.

${ }^{13}$ J. Crank, The Mathematics of Diffusion, 2nd ed. (Oxford University Press, New York, 1975).

${ }^{14}$ H. Carslaw and J. Jaeger, Conduction of Heat in Solids, 2nd ed. (Oxford University Press, New York, 1959).

${ }^{15}$ A. Alonzo, X.-C. Cheng, and T. McGill (unpublished).

${ }^{16}$ R. Naone, E. Hegblom, B. Thibeault, and L. Coldren, Electron. Lett. 33, 300 (1997). 